
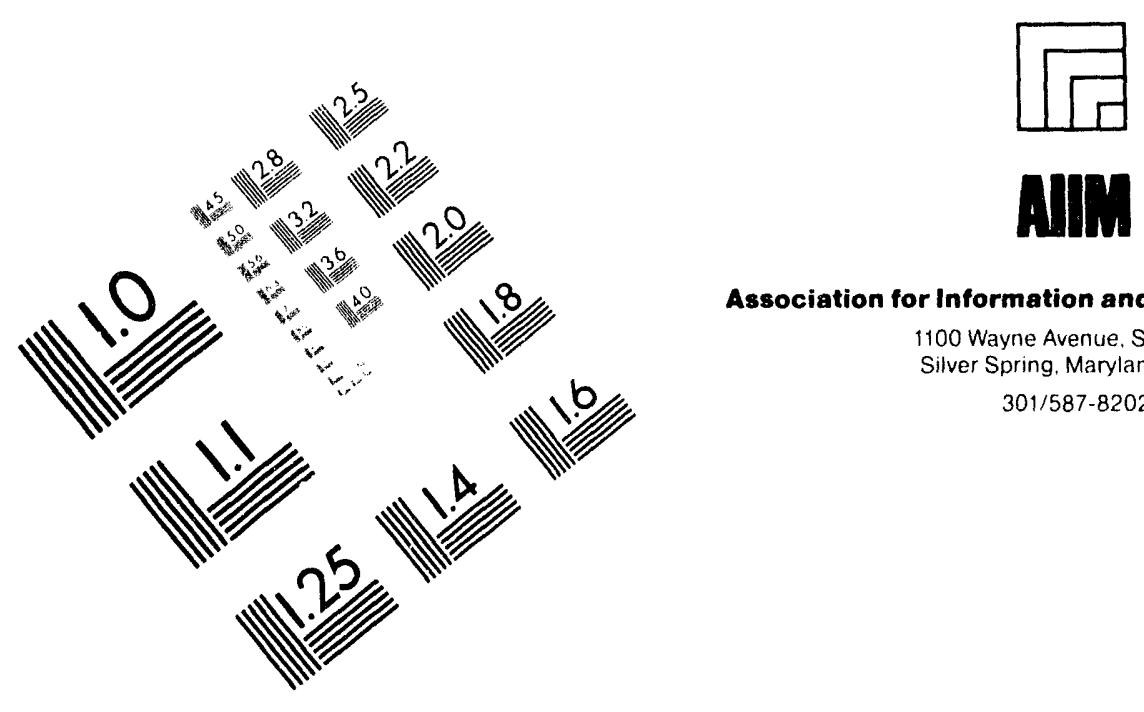

Association for Information and Image Management

1100 Wayne Avenue. Suite 1100

Silver Spring, Maryland 20910

301/587-8202

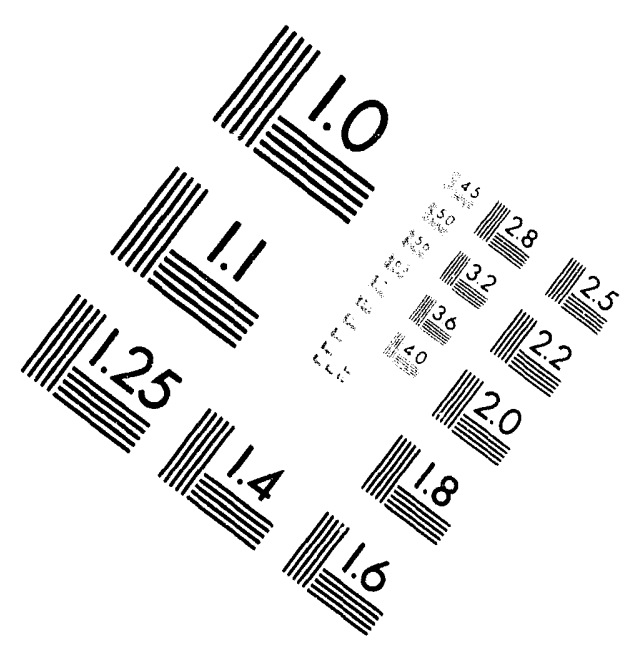

\title{
Centimeter
}

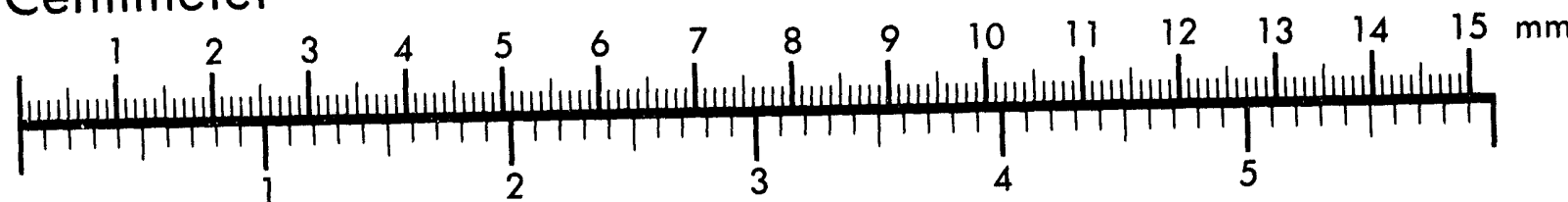

Inches
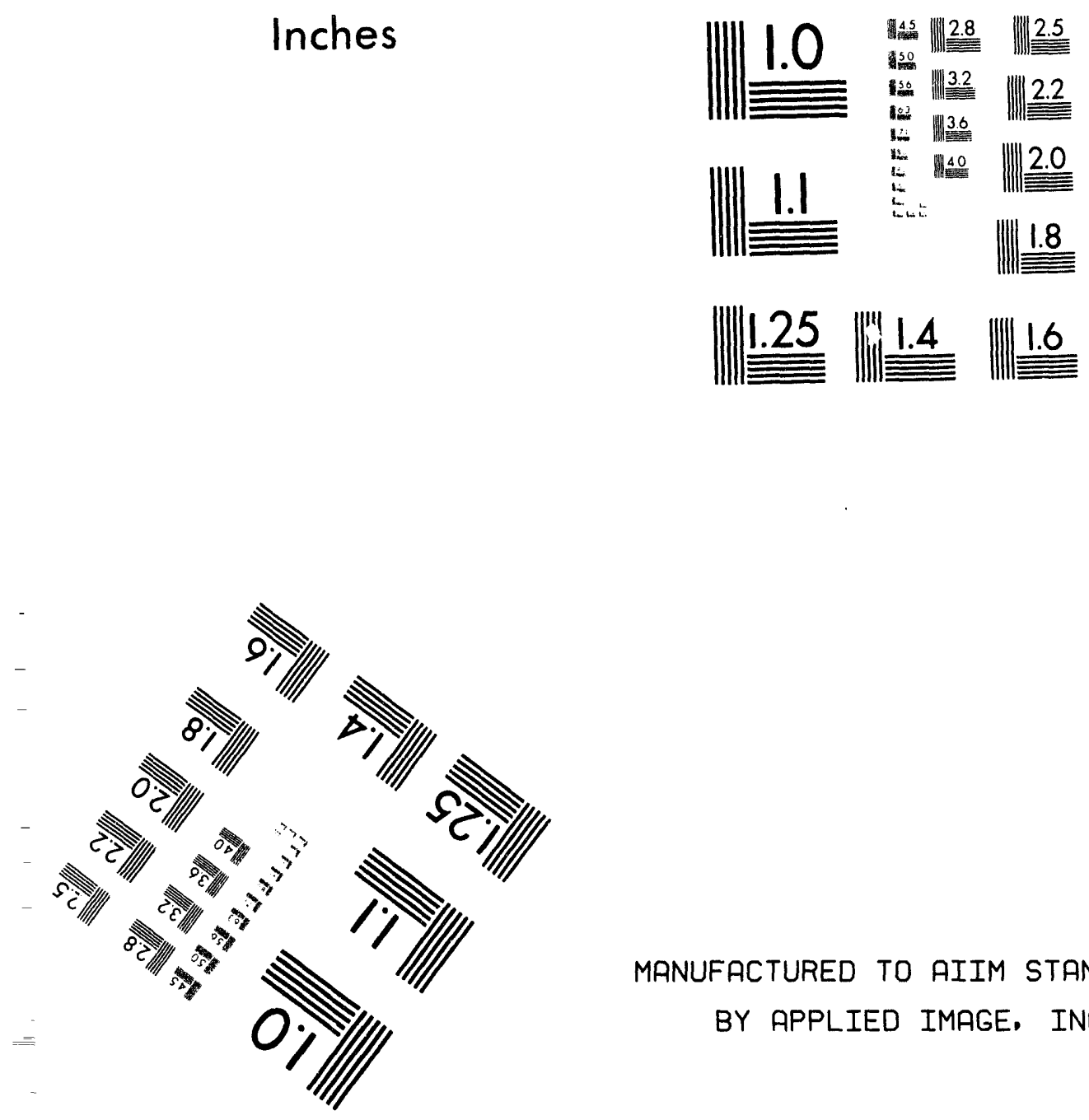

MANUFACTURED TO AIIM STANDARDS

BY APPLIED IMAGE. INC.

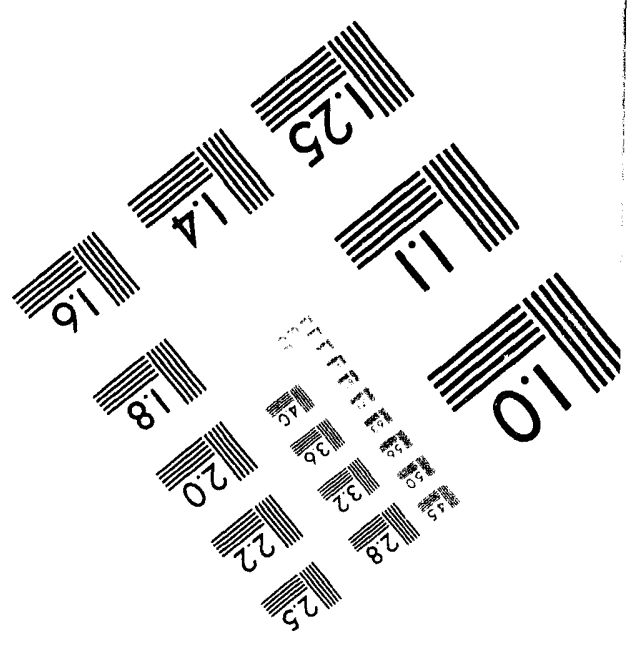



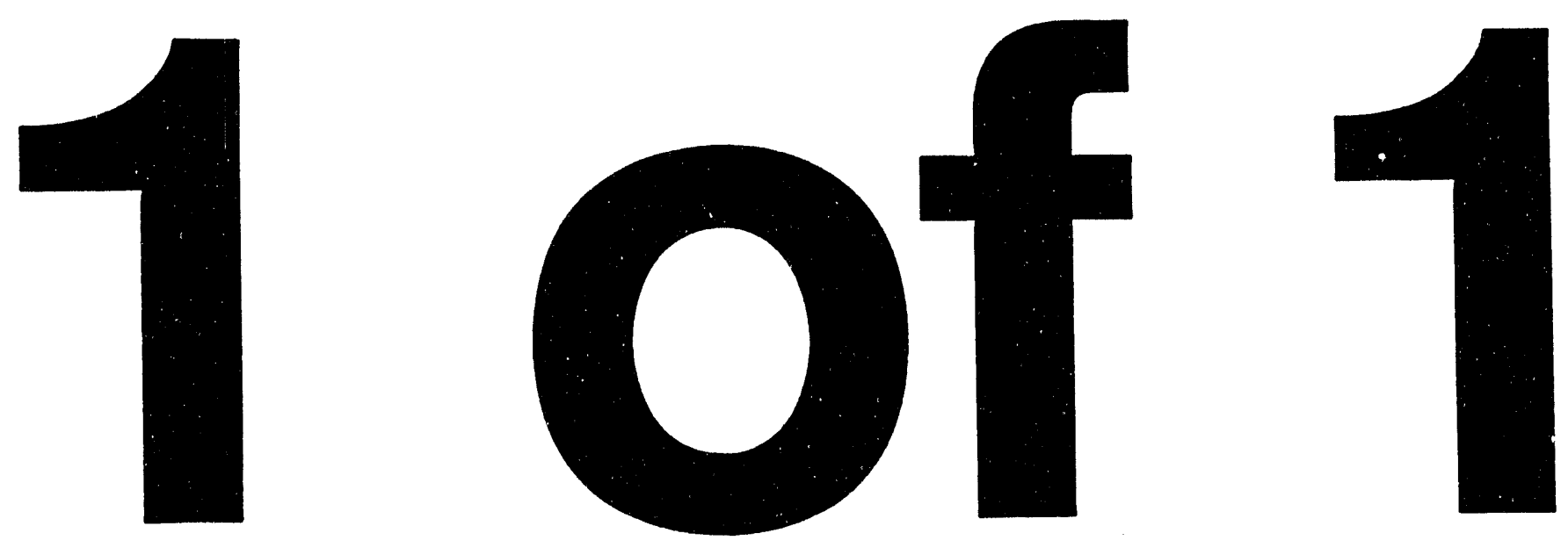


\section{EXTENDED THEORY OF MAIN ION AND IMPURITY ROTATION AND BOOTSTRAP CURRENT IN A SHEAR LAYER}

by

Y.-B. KIM, F.L. HINTON, J. KIM, H. ST. JOHN, T.S. TAYLOR, and D. WRÒBLEWSKI*

This is a preprint of a paper to be presented at the Fourth H-Mode Workshop, November 15-17, 1993, Naka, Japan, and to be printed in a special issue of Plasma Physics and Controlled Fusion.

Work supported by U.S. Department of Energy Grant DE-FG03-92ER54150 and Contracts DE-AC03-89ER51114 and W-7405-ENG-48

*Lawrence Livermore National Laboratory

GENERAL ATOMICS PROJECTS 3938 and 3466 NOVEMBER 1993

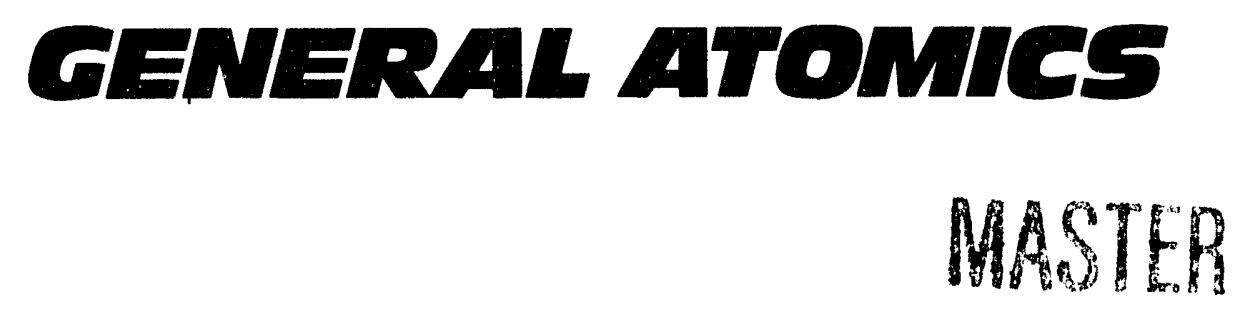




\title{
Extended theory of main ion and impurity rotation and bootstrap current in a shear layer
}

\author{
Y-B Kim, F L Hinton, H St John, T S Taylor and D Wròblewski $\dagger$ \\ General Atomics, San Diego, California, USA
}

\begin{abstract}
In this paper, standard neoclassical theory has been extended into the shear layer. Main ion and impurity ion rotation velocity and bootstrap current within shear layer in $\mathrm{H}$-mode are discussed. Inside the $\mathrm{H}$-mode shear layer, standard neoclassical theory is not valid since the ion poloidal gyroradius becomes comparable to pressure gradient and electric field gradient scale length. To allow for arbitrary ratio of $\rho_{0 i} / L_{n}$ and $\rho_{\theta i} / L_{E n}$, new kinetic theory of main ion species within electric field shear layer has been developed with the assumption that $\rho_{\theta i} / R_{0}$ is still small. As a consequence, both impurity flows and bootstrap current have to be modified. We present modified expressions of impurity flows and bootstrap current are presented neglecting ion temperature gradient. Comparisons with DIII-D measurements are also discussed.
\end{abstract}

\section{Introduction}

Recent experiments from DIII-D and other devices strongly indicate that both fuel and impurity ion flow velocities within flux surface can be described by standard neoclassical theory of rotations except within electric field shear layer very close to the edge (Kim et al., 1993). It is not surprising why neoclassical theory is not supposed to be valid there, since the fundamental assumption of small poloidal gyroradius compared with plasma gradient scale length breaks down. Standard bootstrap current theory also needs modification in a shear layer.

\section{Main ion flow due to orbit squeezing}

The theory of main ion flow within shear layer has been recently developed by Hinton and $\operatorname{Kim}(1993)$. Without assuming small gyroradius ordering (i.e., $\rho_{\theta i} / L_{n}$ and $\rho_{\theta i} / L_{E r}$ are arbitrary), they derived the distribution function within shear layer and parallel and poloidal flows in arbitrary magnetic flux surface geometry. Parallel flow can be written as:

$$
n_{i} u_{\| i}=n_{i} \frac{c I}{B} \frac{\partial \Phi}{\partial \psi}-\frac{B}{\left\langle B^{2}\right\rangle} \frac{c T_{i} I}{e_{i} S} \frac{\partial p}{\partial \psi}-\left(1-\frac{B^{2}}{\left\langle B^{2}\right\rangle}\right) \frac{c I}{e_{i} B} \frac{\partial p_{i}}{\partial \psi}
$$

where the third term is the Pfirsch-Schlüter parallel flow and the orbit squeezing factor $S$ is defined as

\footnotetext{
†Permanent address: Lawrence Livermore National Laboratory, Livermore, California, USA.
} 


$$
S=1+\frac{m c^{2}}{e} \frac{I^{2}}{\left\langle B^{2}\right\rangle} \frac{\partial^{2} \Phi}{\partial \psi^{2}}
$$

Poloidal flow becomes

$$
u_{\mathrm{p}} \equiv \frac{\vec{u} \cdot \vec{B}_{\mathrm{p}}}{B_{\mathrm{p}}}=\frac{B_{\mathrm{p}}}{\left\langle B^{2}\right\rangle} \frac{c I}{n_{i} e_{i}}\left(1-\frac{1}{S}\right) \frac{\partial p_{i}}{\partial \psi}
$$

Note that the poloidal flow velocity is a significant fraction of the ion diamagnetic velocity, when $S \sim 2$ or 3 as in the edge region of DIII-D H-mode and is in reasonable agreement with spectroscopic measurements of the poloidal rotation volocity in helium plasmas in the DIII-D tokamak in $\mathrm{H}$-mode very close to the plasma edge (Kim et al., 1993).

\section{Impurity rotation in the shear layer}

It is useful to derive impurity rotation velocity since most of the rotation measurement can be done only with impurity species. Following the same procedure as in Kim et al. (1991), impurity poloidal rotation velocity without temperature gradient inside shear layer can be expressed as

$$
u_{\theta}^{I}=\frac{1}{2} v_{T_{i} \rho_{i}}\left(-\frac{1}{S L_{p i}}+\frac{Z_{i}}{Z_{I}} \frac{T_{I}}{T_{i}} \frac{1}{L_{p I}}\right) \frac{B B_{t}}{\left\langle B^{2}\right\rangle},
$$

where trace impurity is represented by $I$. Electric field shear reduces main ion pressure gradient contribution to impurity poloidal rotation velocity. Assuming that the first term is still dominant in shear layer, impurity poloidal rotation is still in electron diamagnetic direction as in the case of no electric field shear. Another quantity which can be compared with measurement is the difference between main and impurity local toroidal rotation velocity. Following the standard procedure as in Kim et al. (1991), neglecting the temperature gradient, we obtain .

$$
\Delta V_{\phi} \equiv u_{\phi}^{i}-u_{\phi}^{I}=\frac{1}{2} v_{T i} \rho_{\theta i}\left[-\frac{1}{L_{p i}}+\frac{Z_{i}}{Z_{I}} \frac{T_{I}}{T_{i}} \frac{1}{L_{p I}}\right]\left(1-\frac{I^{2}}{R^{2}\left\langle B^{2}\right\rangle}\right)
$$

Note that the squeezing factor does not appear in this expression, since this difference in toroidal rotation is basically Pfirsch-Schlüter parallel flow. This is expected, since it is the return flow due to the perpendicular ion and impurity diamagnetic flows, which have the same form inside and outside shear layer. Figure 1 shows comparison between theory and measurement in DIII-D He plasma experiment. Measurement is done in the outboard region. One can see excellent agreements between theory and measurement outside shear layer and even inside shear layer.

\section{Bootstrap current in the shear layer}

For the sake of simplicity, we neglect the contribution from impurity species to bootstrap current. The bootstrap current is carried by both ion and electron species at a 


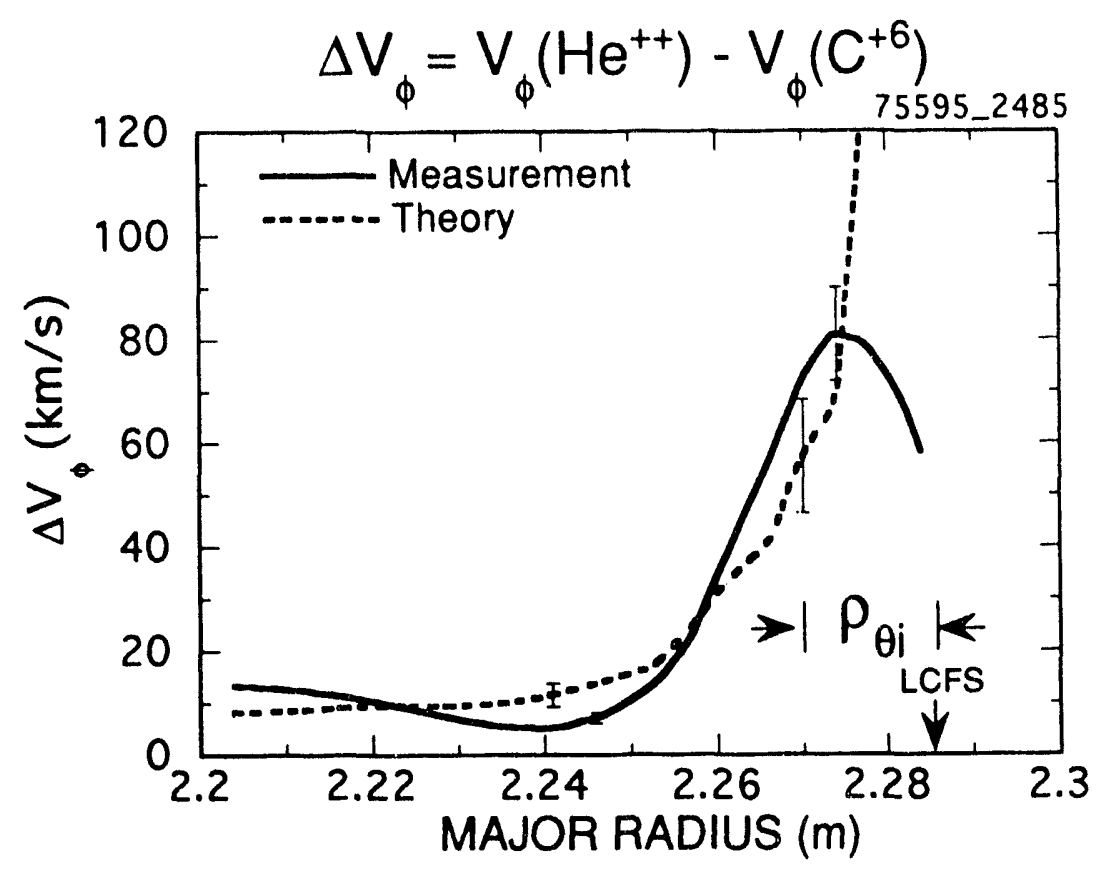

FIG. 1. Toroidal rotation velocity difference between helium main ion and carbon.

roughly equal amount. Within the shear layer, electron dynamics will not be effected by electric field shear because of small electron poloidal gyroradius, but ion dynamics will be modified. If the standard bootstrap current is written as

$$
\left\langle J_{\|} B\right\rangle_{B}=-L_{31}\left(\frac{\partial}{\partial \psi} \ln P_{e}+\frac{\partial}{\partial \psi} \ln P_{i}\right)-L_{32}^{e} \frac{\partial}{\partial \psi} \ln T_{e}-L_{32}^{e} \frac{\partial}{\partial \psi} \ln T_{i}
$$

where the standard bootstrap current coefficients can be found in Hirshman and Sigmar (1981). Note that the last term originates from the standard neoclassical derivation of main ion poloidal rotation. The modified bootstrap current formula can be obtained by replacing this ion temperature gradient driven poloidal rotation with orbit squeezing poloidal rotation. Hence the bootstrap current in shear layer becomes

$$
\left\langle J_{\|} B\right\rangle_{B}=-L_{31}\left(\frac{\partial}{\partial \psi} \ln P_{e}+\frac{1}{S} \frac{\partial}{\partial \psi} \ln P_{i}\right)-L_{32}^{e} \frac{\partial}{\partial \psi} \ln T_{e} .
$$

Note that the ion pressure gradient driven part of bootstrap current is now reduced by $1 / S$. For $S \sim 2$ as in the DIII-D shear layer, we expect that the standard neoclassical theory overestimates bootstrap current by $20 \%$ to $30 \%$. The accurate determination of edge localized bootstrap current is important to determine external magnetohydrodynamic (MHD) stability criteria. Figure 2 shows the comparison between the calculated current profile and the experimental one. The calculated profile is based on the standard neoclassical theory with bootstrap current determined from the finite aspect ratio formula and the experimental profile is reconstructed from MHD equilibrium analysis based on motional Stark effect (MSE), exiernal magnetic probe, 


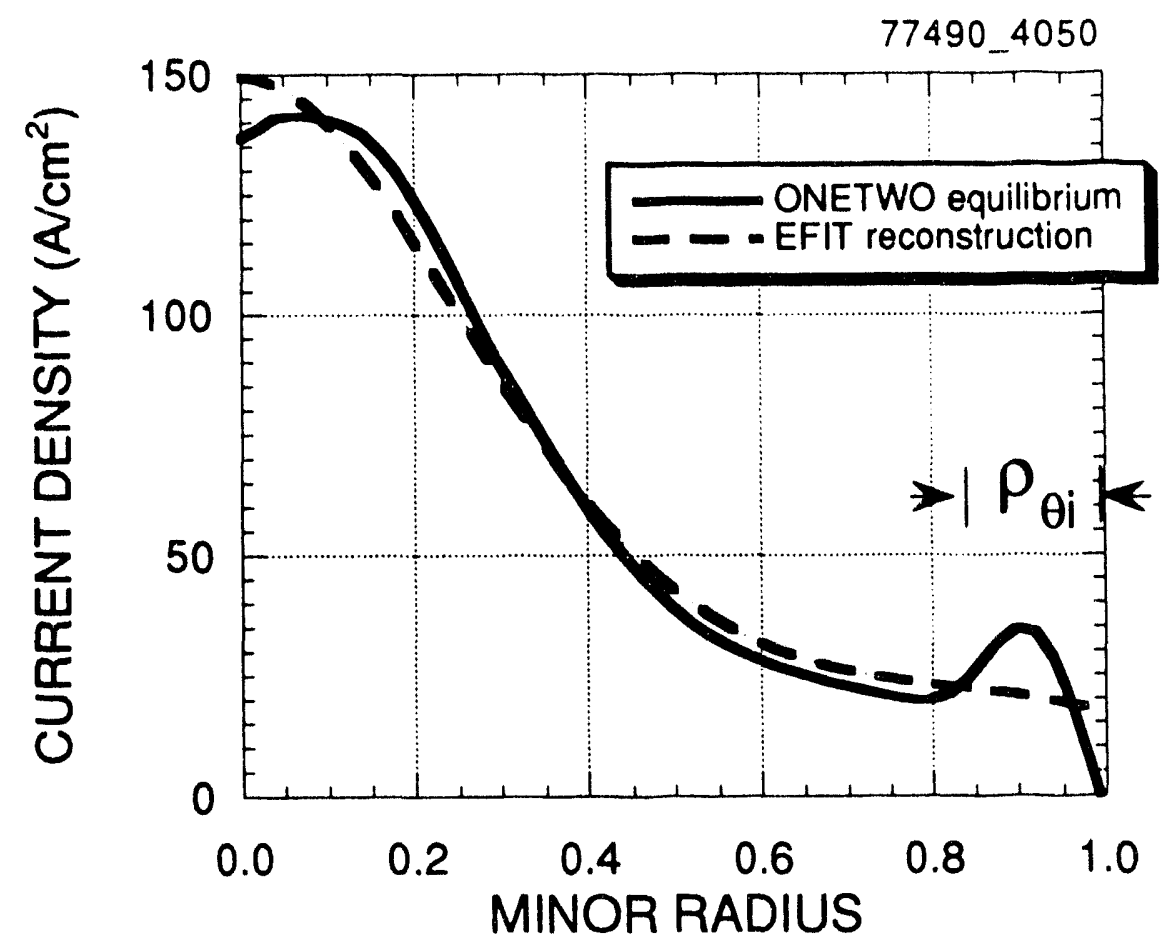

Fig. 2. Current profile comparison between theory (solid line) and experiment (dotted line). The experimental profile is reconstructed from MSE data, external probe data, and kinetic data.

and kinetic data. Within the shear layer, most of the current is bootstrap current due to steep pressure gradient in $\mathrm{H}$-mode. It seems clear that standard bootstrap current theory overestimates experimental measurement by $20 \%$ to $30 \%$.

\section{Conclusions}

The standard neoclassical theories of main ion, impurity ion, and bootstrap current have been extended into the shear layer, taking into account the large electric field shear and steep pressure gradient. When $S>1$, as in the edge region of the most H--mode plasma, the main ion poloidal rotation velocity is in the ion diamagnetic direction with a significant fraction of ion diamagnetic velocity. Impurity poloidal rotation velocity is reduced but still in the electron diamagnetic direction. The difference in main ion and impurity toroidal rotation velocity does not depend on electric field shear. Ion-pressuregradient-driven bootstrap current is also reduced in the shear layer. Experimental results from DIII-D show that orbit squeezing theory is consistent with measurements.

\section{Acknowledgments}

This is a report of work sponsored by the U.S. Department of Energy under Contract No. DE-AC03-89ER51114 and Grant No. DE-FG03-92ER54150; such financial support does not constitute an endorsement by DOE of the views expressed herein. 


\section{References}

Hinton F L and Kim Y-B 1993 submitted to Phys. Fluids B.

Hirshman S P and Sigmar D J 1981 Nucl. Fusion 211079.

Kim J, Burrell K H, Gohil P, Groebner R J, Kim Y-B, St. John H, Seraydarian R P and Wade M 1993 submitted to Phys. Rev. Lett.

Kim Y-B, Diamond P H and Groebner R J 1991 Phys. Fluids B 32050. 

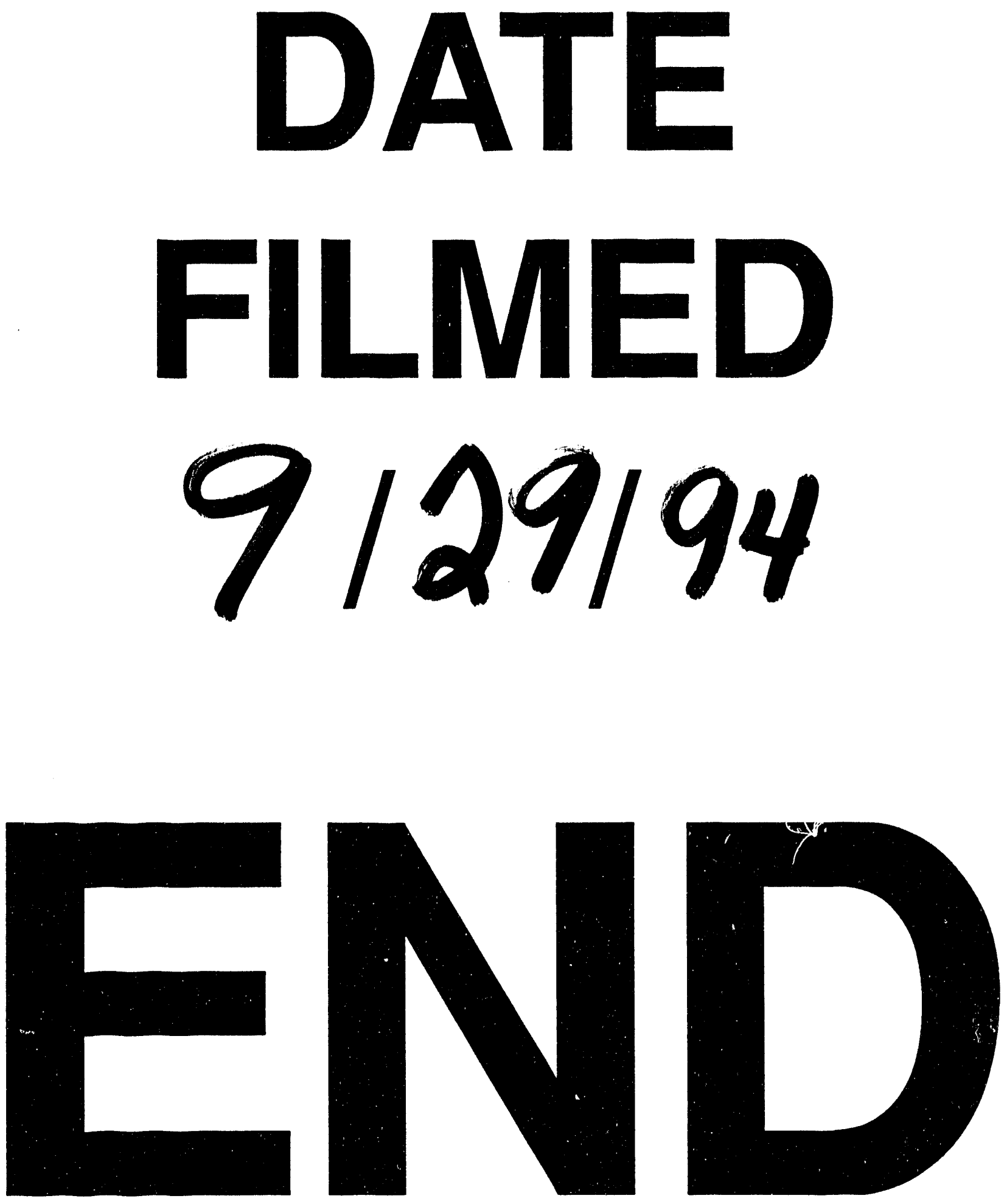
